\title{
Multilayer bandaging plus compression hosiery was better than hosiery alone for unilateral lymphedema of a limb
}

\author{
Badger CM, Peacock JL, Mortimer PS. A randomized, controlled, parallel-group clinical trial comparing multilayer \\ bandaging followed by hosiery versus hosiery alone in the treatment of patients with lymphedema of the limb. Cancer \\ 2000 Jun 15;88:2832-7. \\ QUESTION: In patients with unilateral lymphedema of a limb, is a course of multilayer \\ bandaging (MLB) plus compression hosiery more effective than hosiery alone for \\ reducing limb volume?
}

\section{Design}

Randomised \{allocation concealed*\} $\uparrow$, blinded \{outcome assessors and statisticians $\} \nmid, *$ controlled trial with final follow up at 24 weeks.

\section{Setting}

A lymphedema clinic with hospital sites in London and Surrey, UK.

\section{Patients}

90 consecutive patients who had unilateral upper limb or lower limb lymphedema, with swelling $\geqslant 20 \%$ excess volume over the normal limb. For patients with cancer, at least 12 months had to have elapsed since cancer treatment, with no active disease. Exclusion criteria were paralysis, history of compromised arterial flow in the limb, or limbs too big for hosiery. 83 patients (mean age $57 \mathrm{y}, 92 \%$ women) were included in the analysis; 54 had upper limb lymphedema, and 29 had lower limb lymphedema.

\section{Intervention}

38 patients were allocated to 18 days of MLB: a first layer of a tubular stockinette, with digits bandaged using a retention bandage; the final layers were short stretch extensible bandages. On lower limbs, $\geqslant 2$ layers of bandages were applied in both a spiral and in a figure 8. On upper limbs, bandages were applied in a spiral. Bandages remained in place 24/day and were replaced daily. After 18 days, patients wore hosiery from morning until bedtime for the remainder of the trial. 52 patients were allocated to hosiery alone, beginning on day 1 of the trial. Patients in both groups were given advice on positioning of the swollen limb, exercises to promote drainage, and daily skin care; were taught self massage; and were asked to do all 4 things each day.

\section{Main outcome measures}

Percentage of reduction in excess limb volume ([volume of swollen limb-volume of normal limb]/volume of normal limb) based on an average of individual patient observation periods. Limb volumes were assessed by using an electronic volumeter or by manual surface measurements (circumference ${ }^{2} / \pi$ ).

\section{Main results}

At 24 weeks, patients who received MLB followed by hosiery had greater reductions in limb volume than patients who received hosiery alone (mean percentage of reduction $30 \% v 15 \%$; mean difference $15 \%$, 95\% CI 6 to $24, \mathrm{p}=0.001)$.

\section{Conclusion}

In patients with unilateral lymphedema of an upper or lower limb, a course of multilayer bandaging followed by compression hosiery led to greater percentage reductions in excess limb volume than did hosiery alone.

*See glossary.

†Information provided by author.

\section{COMMENTARY}

Chronic lymphedema is an important problem, particularly in long term survivors of common cancers, including breast cancer. Considerable dogma exists about lymphedema management, but there is a paucity of evidence. This randomised trial by Badger $e t$ al clearly shows the benefits of short term, multilayer bandaging before long term use of compression hosiery. The ease of multilayer bandaging suggests that this intervention could have broad applicability, provided staff are available to apply the bandages.

The findings of Badger $e t$ al differ from those of 5 other recently reported randomised trials of lymphedema. Two studies found no benefit for pneumatic compression over manual lymphatic drainage, ${ }^{1}$ or no treatment ${ }^{2}$; one found no benefit for electrically stimulated lymphatic drainage ${ }^{3}$; one found no added benefit for manual lymphatic drainage over a compression stocking alone ${ }^{4}$; and one found a small benefit for manual lymphatic drainage over compression bandages, particularly for reducing pain. ${ }^{5}$

Although further research is needed in this area, 2-3 weeks of daily application of multilayer bandaging before the use of compression hosiery appears to be useful for patients with chronic limb lymphedema.

Pamela J Goodwin, MD, MSc Mount Sinai Hospital Toronto, Ontario, Canada

1 Johansson K, Lie E, Ekdahl C, et al. A randomized study comparing manual lymph drainage with sequential pneumatic compression for treatment of postoperative arm lymphedema. Lymphology 1998;31:56-64.

2 Dini D, Del Mastro L, Gozza A, et al. The role of pneumatic compression in the treatment of postmastectomy lymphedema. A randomized phase III study. Ann Oncol 1998:9:187-90.

3 Bertelli G, Venturini M, Forno G, et al. Conservative treatment of postmastectomy lymphedema: a controlled, randomized trial. Ann Oncol 1991;2:575-8.

4 Andersen L, Hojris I, Erlandsen M, et al. Treatment of breastcancer-related lymphedema with or without manual lymphatic drainage-a randomized study. Acta Oncol 2000;39:399-405.

5 Johansson K, Albertsson M, Ingvar C, et al. Effects of compression bandaging with or without manual lymph drainage treatment in patients with postoperative arm lymphedema. Lymphology 1999;32:103-10. 